\title{
Liquid Mixing Evaluated Using Entropy in a Lab-on-a-disc Platform
}

\author{
Akinobu Yamaguchi, ${ }^{*}$ Atsushi Ishimoto, Shunya Saegusa, \\ Munehisa Sugiyama, Sho Amano, and Yuichi Utsumi \\ Laboratory of Advanced Science and Technology for Industry (LASTI), University of Hyogo, \\ 3-1-2 Kouto, Kamigori, Ako-gun, Hyogo 678-1205, Japan
}

(Received July 26, 2021; accepted November 24, 2021)

Keywords: lab-on-a-disc, mixing, Euler force, standard deviation, entropy

We present an approach for measuring the mixing degree of two liquids in a microsystem with a mixing mechanism based on the Euler force. By considering Shannon entropy, our proposed method achieves a more reliable evaluation than the conventional technique. The evaluation is performed by analyzing images of the two liquids and calculating the entropy from detected gradation values. We present an accurate analysis of mixing in microfluidics with and without the Euler force. Finally, we confirm that the Shannon entropy used to study the mixing of powders and granules can also be applied to liquid mixing.

\section{Introduction}

Microfluidic systems, long employed in various applications such as integrated point-of-care (POC) devices for early-stage diagnosis, environmental analysis, and chemical reaction systems, are emerging as promising systems for the achievement of the lab-on-a-chip. ${ }^{(1-8)}$ Recently, the lab-on-a-disc (LOD) has attracted much attention owing to its unique features such as simultaneous multi-item inspection, liquid transfer by centrifugal force, isolation from an external liquid transfer mechanism by a confinement system, and suppression of waste liquid by this isolation. ${ }^{(9-18)}$ These multiple superior features of LODs can be used in commercial immunoassay systems. ${ }^{(19-23)}$ Diagnostic devices using LODs have already been released by some companies such as Roche, ${ }^{(20)}$ Abaxis, ${ }^{(21)}$ Samsung Healthcare, ${ }^{(22)}$ and Sysmex. ${ }^{(23)}$ For example, Cobas blOl released by Roche can measure biomarkers, HbAlc, and lipids. Recently, Sysmex has developed a novel compact immunoassay system that can achieve a limit of quantification (LoQ) of $3.6 \mathrm{pg} / \mathrm{mL}$ for the biomarker PSA of prostate cancer. In the Sysmex LOD systems, such high sensitivity can be achieved by the combination of magnetic-beads-based immunoassay, mixing using the Euler force, and the lavage mechanism by separating the lavage space from the assay space.

In an integrated microchannel, the Reynolds number becomes so small that it is difficult to mix different liquids. Mixing is an essential step in promoting a chemical reaction. In the LOD

*Corresponding author: e-mail: yamaguti@lasti.u-hyogo.ac.jp

https://doi.org/10.18494/SAM.2021.3567 
system developed by Sysmex described above, ${ }^{(23)}$ high mixing efficiency is realized by using fine magnetic particles and the Euler force. In the research reports from other groups, the sensitivity of the assay is strongly influenced by the mixing process and procedure. Quantifying mixing is essential for optimizing various industrial processes and laboratory chemical reactions. It is crucial at both the macroscopic scale and the microscopic level when dealing with microfluidics in lab-on-a-chip platforms and micro total analysis systems. Moreover, liquid mixing is one of the main chemical operations in the construction of chemical reaction systems. How should we quantitatively evaluate how the mixing is progressing, how efficient it is, and so forth?

Quantitative evaluation of liquid mixing typically uses an approach based on the standard deviation. ${ }^{(16,18)}$ This method is known as Lacey mixing evaluation and is often used to determine the mixing degree of powders and granules. ${ }^{(24)}$ The technique compares the statistical distribution of measured data with the statistical variances of perfectly mixed and completely separated states. However, because it is a statistical process, its reliability is low when the sample size is small. Moreover, it cannot be used when a segregation phenomenon occurs that prevents mixing and dispersal. ${ }^{(25)}$ In particular, mixing in a microchannel tends to induce such a phenomenon because the Reynolds number is low, and mixing is difficult to achieve in a laminar flow state. Therefore, some previous studies concluded that the absolute mixing degree cannot be determined using Lacey mixing evaluation. ${ }^{(25)}$

In this study, we propose an approach based on Shannon informational entropy to evaluate mixing in a microchannel of a LOD platform. ${ }^{(16)}$ The Shannon entropy is uniquely determined regardless of the initial state, ${ }^{(26,27)}$ and it provides a rigorous measure of system homogeneity. As is well known, entropy quantitatively indicates disorder. The entropy is low for an ordered state, and it increases as mixing occurs and the state becomes disordered. We calculate the system entropy using the Shannon entropy from the gradation values of each pixel in continuously shot images, and we confirm the correlation between time and entropy. The specific calculation method and evaluation results are discussed. We show that our technique provides a mathematically supported way to evaluate mixing in a microchannel regardless of the presence or absence of segregation phenomena.

\section{Basic Idea and Analysis Method}

For powder mixing, the entropy is determined from the number of particles dispersed in a unit observation area. Given the similarity between powder mixing and liquid mixing, we considered that liquid mixing corresponds to a mapping of the color and contrast of an image of unit area. To evaluate the mixing degree, we obtained the Shannon entropy from an image of the microchamber where the mixing occurred. According to previous works, under the assumption of particle mixing, the system is divided into bins, and the number $n_{j, c}$ of particles of species $c$ $(c=1,2,3,4, \ldots, C)$ is numbered for each $\operatorname{bin}(j=1,2,3,4, \ldots, M)$. Then, the system entropy $S$ is given by ${ }^{(25-27)}$ 


$$
S=-\sum_{j=1}^{M} \sum_{c=1}^{C} p_{j, c} \ln p_{j, c},
$$

where $p_{j . c}$ is the joint probability of finding a group/complex of $P_{c}$ particles of species $c$ in $\operatorname{bin} j$ :

$$
p_{j, c}=\frac{\frac{n_{j, c}}{P_{c}}}{\sum_{j=1}^{M} \sum_{c=1}^{C} \frac{n_{j, c}}{P_{c}}} .
$$

In our study, the equivalent of a particle is a safranin molecule. The equivalent of a bin is a pixel. Depending on the number of safranin molecules per bin, the color strength per pixel corresponds to the probability of a particle being present. To simplify and calculate the entropy, we divided the system into pieces and labeled each piece with a number $j(j=1,2,3, \ldots n)$. Each piece corresponds to a pixel of the obtained image. We assumed that fine particles were distributed in each piece and estimated the distribution probability. This distribution probability corresponds to the pixel gradation, and such logic can be directly applied to the mixing of different liquids. That is, for each pixel, the gradation can be defined as the degree of mixing. Normalizing the gradation for each pixel corresponds to the probability $p_{j}(j=1,2,3, \ldots)$ of mixing. The entropy for each pixel is given by $p_{j} \ln p_{j}$. We used the Shannon entropy $S$ instead of the conventional evaluation based on the standard deviation because it enables stable absolute evaluation. $S$ represents the information entropy for a particular experimental condition with a set of $M$ possible outcomes. It can be expressed as the total entropy for the entire evaluation area. Therefore, $S$ is given by

$$
S=-\sum_{j=1}^{M} p_{j} \ln p_{j}
$$

where $p_{j}$ is the probability of a particular gradation value for pixel $j$.

We performed a series of procedures in our Shannon entropy analysis. First, to make the measurement area the same on a pixel-by-pixel basis, we corrected the position of the image taken by the observation system. In this system, a strobe flash was synchronized with a charge-coupled device (CCD) camera that had a resolution of 96 dpi and a frame rate of about 30 fps. For position-drift correction, we used Register Virtual Stack Slices, a plug-in function of the image-processing software Fiji Image. ${ }^{(28)}$ Next, we trimmed the measurement area and converted it to 8-bit grayscale. We then normalized the gradation value for each pixel in the measurement area (the intensity is given by an integer from 0 to 255) by dividing by the maximum value and converting the value to a probability. Finally, we calculated the Shannon entropy using Eq. (3). 
We used the Euler force to mix the liquids. This force produces acceleration and deceleration and is proportional to the time derivative of the angular velocity vector $\omega$ as follows: ${ }^{(11,16,18)}$

$$
\boldsymbol{F}_{\text {Euler }}=-m \frac{d \boldsymbol{\omega}}{d t} \times \boldsymbol{r},
$$

where $m$ is the mass and $\boldsymbol{r}$ is the distance vector from the center of a rotating coordinate system to the mass.

\section{Experiments}

\subsection{Device fabrication}

First, a device for a Euler force mixing experiment was prepared by joining polydimethylsiloxane (PDMS) and polymethylmethacrylate (PMMA) substrates (Cell-Cast Acrylic sheet, Nitto Jushi Kogyo Co., Ltd.). As schematically illustrated in Fig. 1, the fabrication process was performed. The fabrication procedure is described as follows:

(1) To fabricate the shapes of the reservoir and mixing chamber on the PMMA substrate (A), deep X-ray lithography (DXL) was performed at BL11 of the New SUBARU synchrotron facility in the University of Hyogo. This fabricated PMMA structure was the mold for PDMS.

(a)

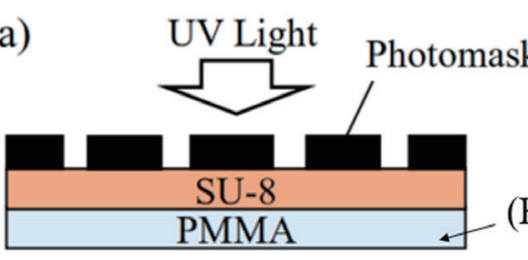

(b)

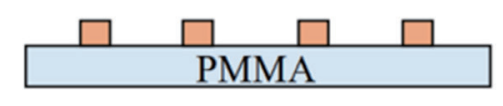

(c)

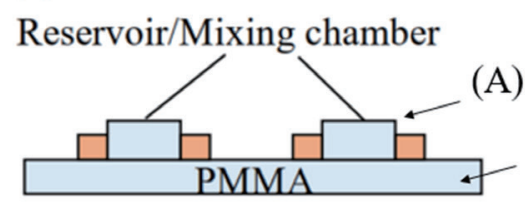

(d)

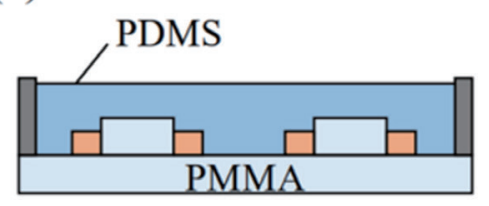

Heating on hotplate

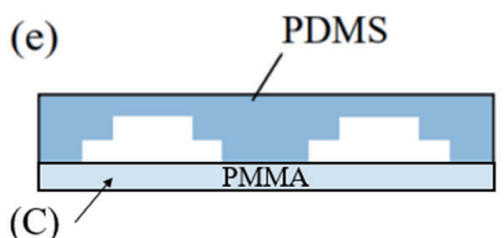

Fig. 1. (Color online) Schematic diagrams of fabrication of a device. (a) UV lithography process performed on PMMA substrate (B). (b) Schematic cross-sectional view of device after developing SU-8. (c) Schematic crosssectional view of master mold consisting of SU-8 and PMMA. The PMMA substrate (A), which was processed by DXL in advance, was joined to the PMMA substrate (B). (d) PDMS molding process using the master mold. (e) Schematic cross-sectional view of completed device consisting of reservoir, mixing chamber, and capillary valve. Finally, the device used for the experiment was completed by drilling vent holes in the device. 
(2) A master mold for molding PDMS was prepared on the PMMA substrate (B) using UV lithography and SU-8 resist as shown in Figs. 1(a) and 1(b).

(3) The PMMA substrate (A) with the shapes of the reservoir and mixing chamber was joined to the PMMA substrate (B) integrated with SU-8 patterns. This bonding was performed by adding methyl methacrylate to the bonding surface and heating at $50{ }^{\circ} \mathrm{C}$ for $10 \mathrm{~s}$. This combined PMMA + SU-8 structure (A+B) was the master mold as shown in Fig. 1(c).

(4) After that, a metal frame was attached to the bonded PMMA substrate, and a PDMS (SILPOT 184, Dow Corning Toray Co. Ltd., Japan) precursor (a liquid in which a PDMS monomer and a cross-linking agent were mixed at a ratio of 10:1) was poured into the bonded PMMA substrate at $50{ }^{\circ} \mathrm{C}$ and heated on a hot plate for $6 \mathrm{~h}$ as shown in Fig. 1(d). After that, the PDMS was peeled off from the mold.

(5) Finally, the peeled PDMS was bonded to the other flat PMMA plate (C) to complete the device as shown in Fig. 1(e).

\subsection{Experimental procedure}

In the experiment, we used colored water, in which $0.1 \mathrm{wt} \%$ safranin solution (FUJIFILM Wako Pure Chemical Corp.) was added to pure water, and a concentrated aqueous solution of phosphoric acid. We inserted the colored water into a reservoir in the disc and added the solution of phosphoric acid to a mixing chamber as schematically illustrated in Fig. 2. To evaluate the mixing of these two liquids, we performed time-resolved real-space observation using a

(a)

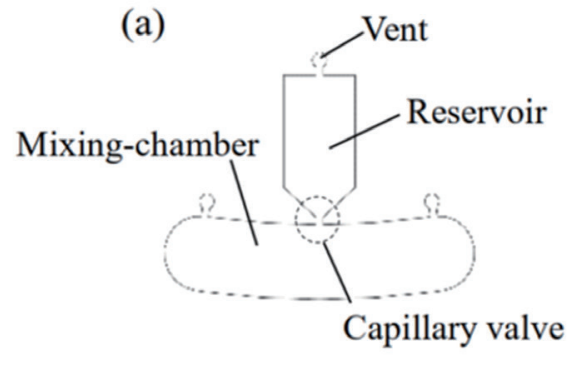

(c)

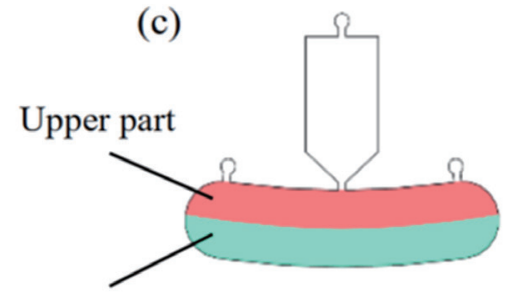

Lower part (b) Lower density liquid

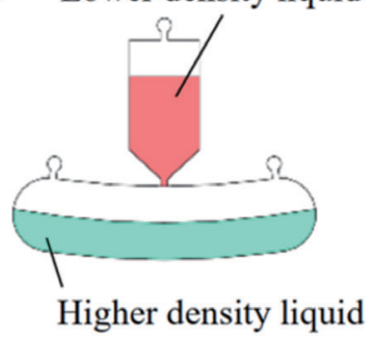

(d)

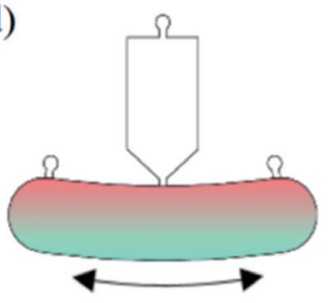

Acceleration / Deceleration

Fig. 2. (Color online) Schematics of (a) mixing chamber structure consisting of vent, reservoir, mixing chamber, and capillary valve, (b) initial stage of mixing chamber before breaking capillary valve, (c) mixing chamber just after injection of lower density liquid, and (d) mixing proceeding by Euler force accompanied with acceleration and deceleration. 
laboratory-built spin-stand experimental apparatus. ${ }^{(2)}$ We handled and collected the images of the mixed liquids using the spin-frequency protocol depicted in Fig. 3.

First, the demonstration device was rotated with an angular acceleration of $10.43 \mathrm{rad} / \mathrm{s}^{2}$ to break a capillary valve and insert colored water into the mixing chamber. This state was the initial state. Then, the disc was decelerated and accelerated at a constant angular acceleration, and the rotation speed was set to $16.66 \mathrm{~Hz}$. After reaching this speed, an increase and decrease in the rotation speed of $16.66 \mathrm{~Hz}$ occurred every $2 \mathrm{~s}$ until $280 \mathrm{~s}$, as illustrated in Fig. 3. This operation of repeated angular acceleration and deceleration generated the Euler force employed for mixing. In this study, we verified that the Euler force induced mixing by varying the specific density difference $d(\%)$ between the aqueous solution of phosphoric acid and the colored water. Additionally, we varied the angular acceleration $\alpha\left(\mathrm{rad} / \mathrm{s}^{2}\right)$ of the device and the depth $D(\mathrm{~mm})$ of the mixing chamber. The length $L$ and width $w$ of the chamber were 20 and $2.0 \mathrm{~mm}$, respectively. We compared cases when $D$ was 0.50 and $0.80 \mathrm{~mm}$ under the conditions of $\alpha=52.35 \mathrm{rad} / \mathrm{s}^{2}$ and $d=1 \%$.

\section{Results and Discussion}

Figure 4 shows a series of observation time dependences of the mixing chamber (a)-(c) with and (d)-(f) without the Euler force. In the absence of the Euler force, the protocol shown in Fig. 3 was absent and the stopped state was maintained after the capillary valve was broken and liquid was injected into the mixing chamber. That is, mixing proceeded by diffusion including gravity and inertia. As a result, even when the evolution time reached $280 \mathrm{~s}$, part of the mixing liquid was not completely mixed as shown in Fig. 4(f). On the other hand, when the Euler force, shown

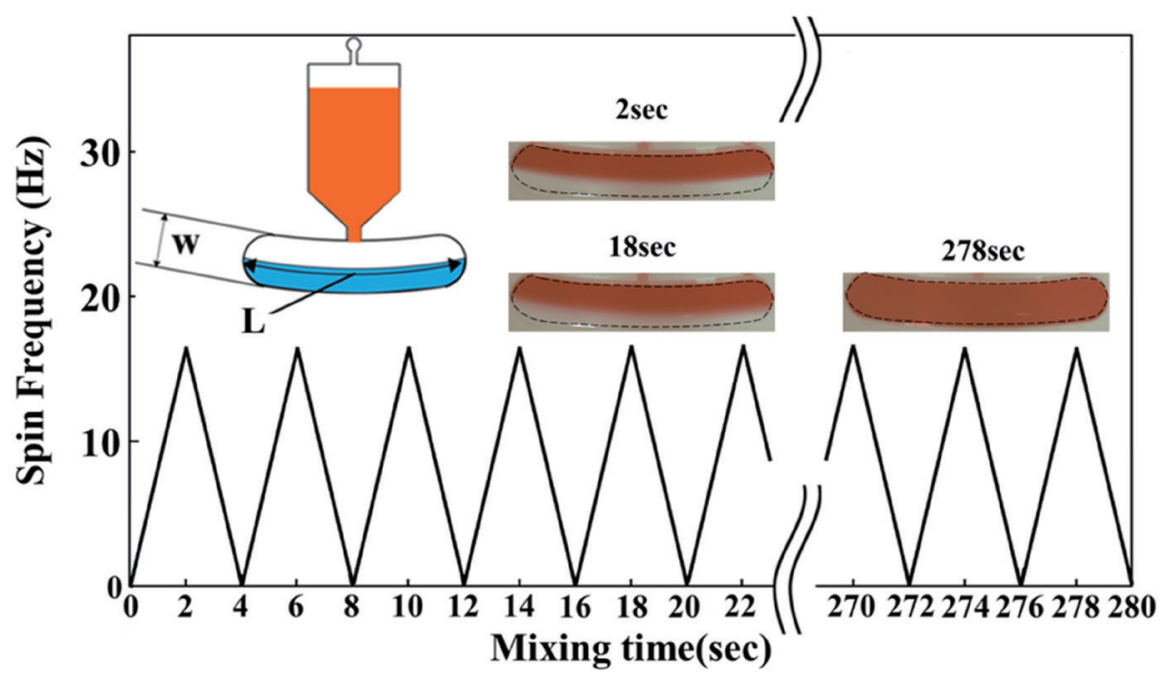

Fig. 3. (Color online) Spin protocol and mixing performance of LOD. After the injection of orange-colored liquid into the mixing chamber, reciprocating and mixing excited by the Euler force due to the modulation of the spin frequency were performed for $280 \mathrm{~s}$. Acceleration and deceleration were repeated every $4 \mathrm{~s}$. The inset diagram of the mixing chamber depicts the typical characteristic dimensions. Optical observation results of the mixing chamber at 2,18 , and $278 \mathrm{~s}$ are shown in the inset images. 

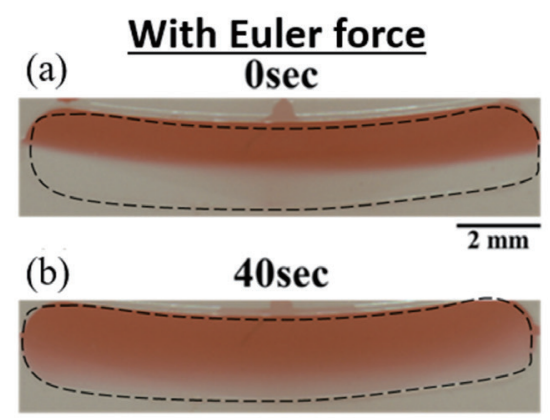

(c)

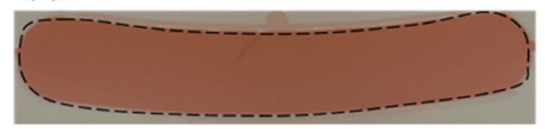

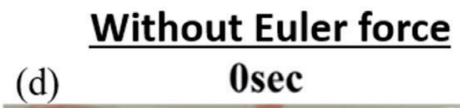

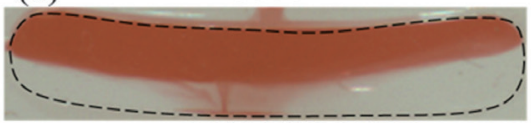

(e) 40sec

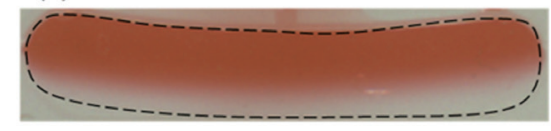

(f) 280sec

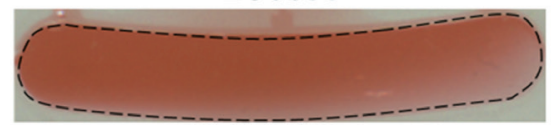

Fig. 4. (Color online) Successive images of the mixing chamber after (a, d) 0, (b, e) 40, and (c, f) $280 \mathrm{~s}$. The angular acceleration is $52.35 \mathrm{rad} / \mathrm{s} 2$, the depth of the chamber is $0.80 \mathrm{~mm}$, and the density difference is $1 \%$. The images in (a)-(c) and (d)-(f) were obtained with and without the Euler force, respectively.

in the protocol of Fig. 3, was applied, it can be seen that the mixing progressed with time. Successive images of the chamber after (a) 0, (b) 40, and (c) $280 \mathrm{~s}$ with $D=0.80 \mathrm{~mm}$ during the mixing protocol with the Euler force are shown in Fig. 3. Just after the colored liquid was introduced into the mixing chamber by breaking the valve, the liquid phases were clearly separated, as shown in Fig. 4(a). As the mixing time increased, the boundary between the two liquids became unclear, and the color of the liquids in the chamber became uniform after $280 \mathrm{~s}$, as shown in Fig. 4(c).

Here, to confirm the problem in the conventional mixing evaluation method using Lacey mixing evaluation, we plot the standard deviation as a function of mixing time in Figs. 5(a)-5(d). Before describing the evaluation of the mixing ratio using Shannon entropy, we estimate the mixing using the conventional standard deviation method to compare the Shannon entropy evaluation with the standard deviation evaluation. Figure 5 shows the mixing time dependence of the standard deviation. This time dependence of the standard deviation basically shows that the standard deviation tends to decrease with time. This is because when the two liquids are clearly separated, the dispersion is large, and when the two liquids are completely mixed, the dispersion is the minimum. The results of changing the strength of the Euler force by preparing density differences of 1 and $10 \%$ and chamber lengths of $D=0.50$ and $0.80 \mathrm{~mm}$ are summarized in Fig. 5. As can be seen from the results, there is a region where the standard deviation does not depend on time [the ellipses in Figs. 5(b) and 5(c)] under the conditions of mixing by the Euler force, $d=10 \%, D=0.50 \mathrm{~mm}$, and $\alpha=52.35 \mathrm{rad} / \mathrm{s}^{2}$. This means that the mixing has progressed sufficiently or that the method using the standard deviation does not give a correct evaluation. Here, these conditions $\left(d=10 \%, D=0.50 \mathrm{~mm}\right.$, and $\left.\alpha=52.35 \mathrm{rad} / \mathrm{s}^{2}\right)$ are those with the strongest Euler force in this experiment, and there is a possibility that the mixture is sufficiently mixed and saturated. However, the standard deviation should be near zero when the mixing is complete, but it did not actually reach zero. This result indicates that the method using this standard 

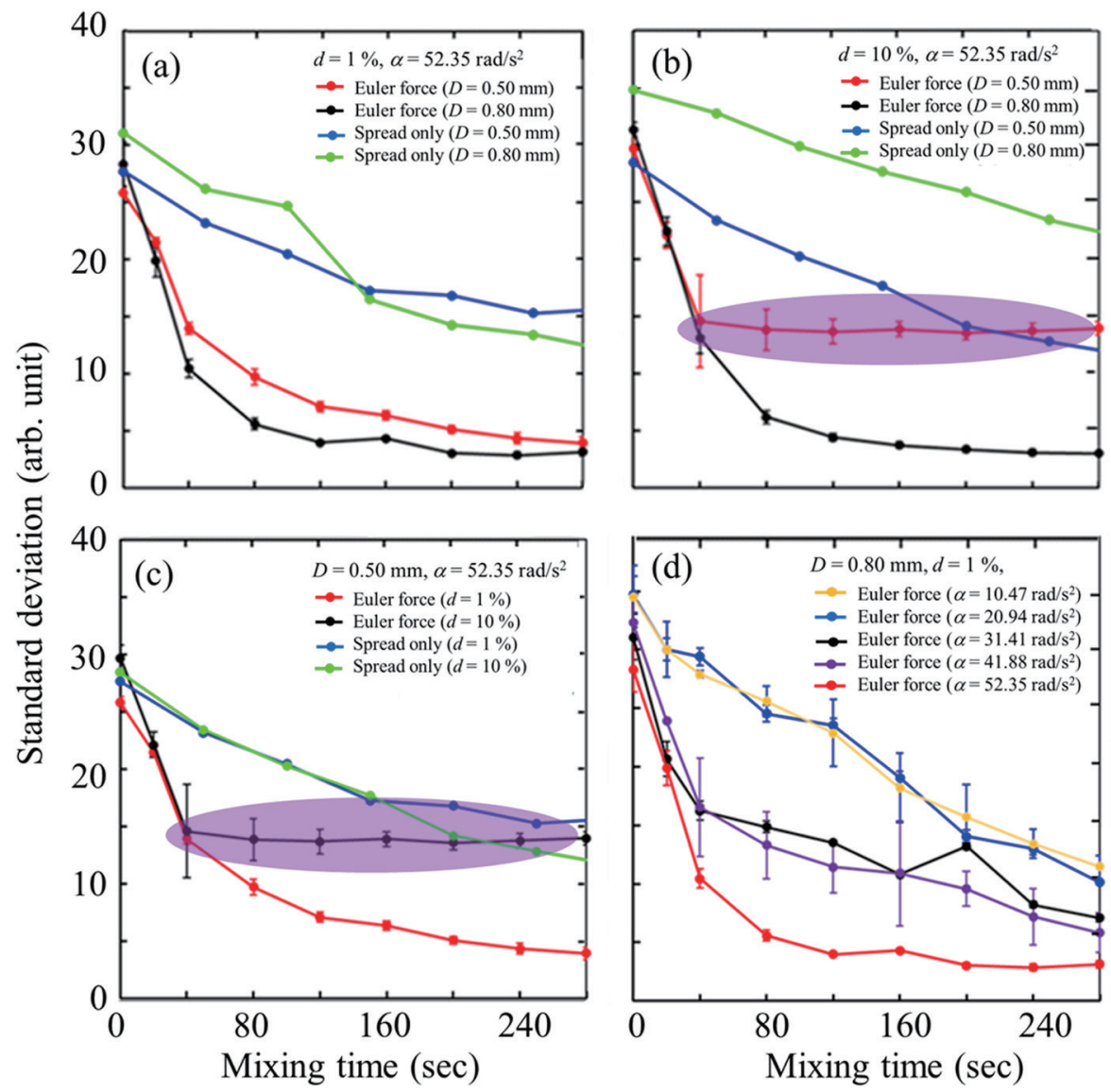

Fig. 5. (Color online) Summaries of conventional standard deviation evaluation of mixing. (a) Depth of mixing chamber varied for liquid density difference $d$ of $1 \%$ and angular acceleration $\alpha$ of $52.35 \mathrm{rad} / \mathrm{s}^{2}$. (b, c) Chamber depth $D$ varied for $d=10$ and $1 \%$, respectively, with $\alpha$ fixed at $52.35 \mathrm{rad} / \mathrm{s}^{2}$. (d) Angular acceleration dependence of mixing for $D=0.80 \mathrm{~mm}$ and $d=1 \%$.

deviation may have failed in some way. In addition, in the other cases, the standard deviation did not saturate and reach zero even when sufficient time had passed and the mixing was complete.

Next, we introduce the time evolution of the mixing evaluation using the Shannon entropy given by Eq. (3). By describing the time evolution of the Shannon entropy, we have introduced an evaluation method that provides a meaningful physical quantity of the mixing degree in a microchannel. Moreover, we have used our approach to analyze the time evolution of twocomponent mixing induced by the Euler force in a lab-on-a-chip platform. In the following, we present a systematic investigation of how various parameters (such as chamber depth, angular acceleration, and density difference between the liquids) affect this time evolution. Moreover, to determine whether mixing by the Euler force itself is effective, we compare cases in which mixing was achieved with and without this force.

Figure 6 shows how the entropy depends on the mixing time. For mixing induced by the Euler force, the entropy is normalized with its value when mixing is complete (at $280 \mathrm{~s}$ ). For the case of no Euler force, the value at $34 \mathrm{~min}$ is used. As shown in Fig. 6, the entropy increases in 

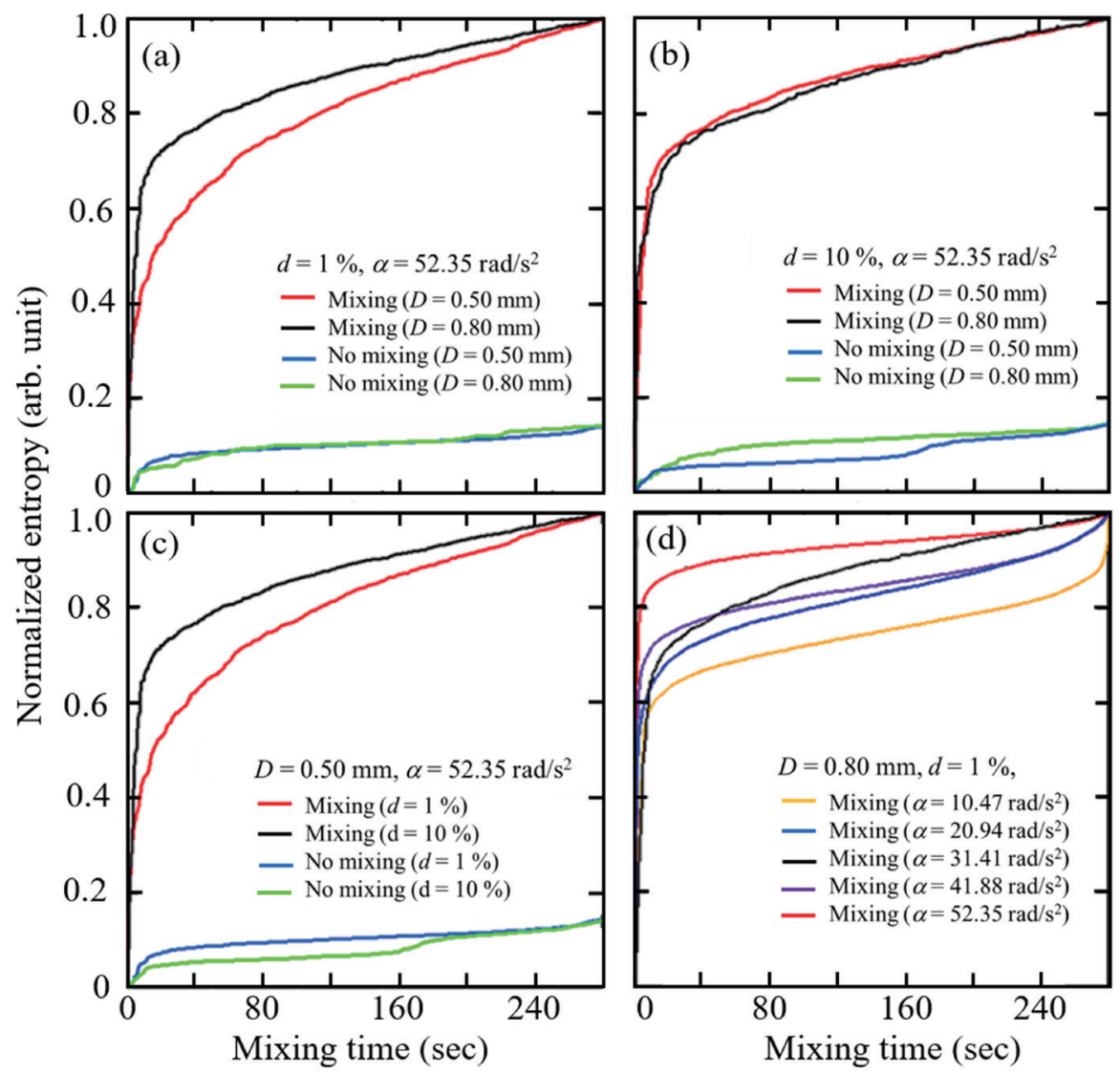

Fig. 6. (Color online) Normalized entropy versus mixing time. (a) Depth of mixing chamber varied for liquid density difference $d$ of $1 \%$. (b) Chamber depth $D$ varied for $d=10 \%$. (c) Liquid density difference varied with parameters $d, D$, and angular acceleration $\alpha$ fixed. (d) $D$ varied and $d$ and $D$ fixed.

all systems over time. This finding suggests that the mixing proceeds by the Euler force or diffusion and is consistent with the second law of thermodynamics. Figures 6(a) and 6(b) show the mixing properties for $d=1$ and 10\%, respectively. Figure 6(c) presents the impact of $\mathrm{d}$ on $S$ for $D=0.50 \mathrm{~mm}$ and $\alpha=52.35 \mathrm{rad} / \mathrm{s}^{2}$. Finally, Fig. 6(d) depicts the effect of $\alpha$ on $S$ for $D=0.80$ $\mathrm{mm}$ and $d=1 \%$. As shown in Fig. 6(a), the entropy for $D=0.80 \mathrm{~mm}$ and Euler force mixing is always higher than that for the other cases. The reason for this trend is that the Reynolds number of the device with $D=0.80 \mathrm{~mm}$ is larger, making turbulence more likely to occur. As a result, the mixing is promoted in a device with a larger Reynolds number. By contrast, no significant difference in the entropy time evolution is observed in Fig. 6(b) when $D$ is varied. Such behavior is caused by a centrifugation effect resulting from the relatively large density difference. This effect becomes dominant and mixing proceeds only by diffusion rather than by the Euler force. In Fig. 6(d), the entropy is always larger for $\alpha=52.35 \mathrm{rad} / \mathrm{s}^{2}$ than for $\alpha=26.17 \mathrm{rad} / \mathrm{s}^{2}$. As shown by Eq. (4), the Euler force is proportional to the angular acceleration. Therefore, this result indicates that the Euler force significantly contributes to the mixing process.

Here, we consider the evolution of entropy with the mixing time. When the two liquids are mixed, safranin, which is the source of the color, is scattered and diffused in the liquid solution. 
That is, by considering the dispersion of safranin molecules, it can be inferred that the time evolution of entropy can be described by the scattering relaxation time model. The normalized entropy $S_{N}$ is written as

$$
S_{N}=1-\exp \left(\frac{-t}{\tau}\right)
$$

where $\tau$ is the relaxation time. Below, the relaxation time was calculated by fitting the time dependence of the entropy in Fig. 6 using Eq. (5). The reciprocal of the calculated scattering relaxation time, that is, the scattering frequency, plotted in Fig. 7 as a function of the angular acceleration shows that the larger the angular acceleration (the stronger the Euler force), the higher the scattering frequency. This is because the greater the Euler force, the greater the rate of collision and dispersion per unit time. That is, the scattering frequency increases as the Euler force increases. When this behavior is fitted with the following polynomial function:

$$
\frac{1}{\tau}=K_{0}+K_{1} \cdot \frac{d \boldsymbol{\omega}}{d t}+K_{2} \cdot\left(\frac{d \boldsymbol{\omega}}{d t}\right)^{2},
$$

where $K_{i}(i=0,1,2)$ are coefficients, it can be seen that the agreement is very good. Here, $K_{0}=0.0042965 \pm 0.00102\left(\mathrm{~s}^{-1}\right), K_{1}=-5.7196 \times 10^{-5}\left(\mathrm{rad}^{-1}\right)$, and $K_{2}=6.653 \times 10^{-6}\left(\mathrm{~s}^{-\mathrm{rad}^{-2}}\right)$. The coefficient $K_{0}$ originates from various factors such as Brownian scattering and measurement systems. The coefficient $K_{1}$ corresponds to the resistance of the molecule to moving through the liquid owing to its viscosity, similar to the raindrop model. The coefficient $K_{2}$ corresponds to the quadratic term, since the second-order proportional component behaves in accordance with the protocol shown in Fig. 3 in which the Euler force reverses after a certain period of time. The

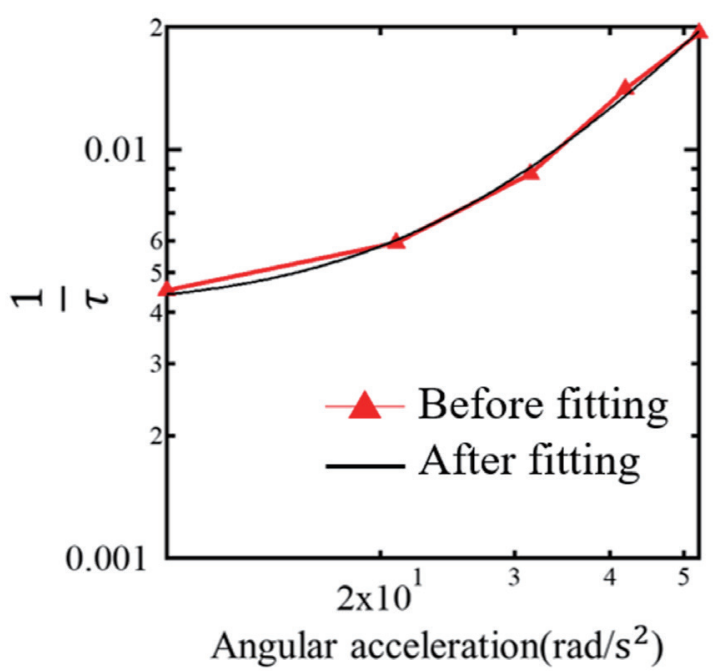

Fig. 7. (Color online) Scattering frequency $1 / \tau$ as a function of angular acceleration. The red solid triangles denote the evaluation values from Fig. 6. The black solid line corresponds to the fitting curve described by Eq. (6). 
action of forcibly rotating the forward rotating state in the reverse of the direction of rotation is added. That is, the two liquids containing the molecules collide with each other twice on the way back and forth. In other words, a nonlinear mixing or scattering process occurs with the generation of turbulence. Thus, the evaluation of the system entropy can provide the details of the time evolution of the mixing properties in the microchamber under the application of the Euler force.

\section{Conclusion}

In conclusion, our proposed method serves as a general technique for the quantitative evaluation of liquid mixing. In particular, it can be used to evaluate mixing processes in lab-ona-chip platforms and in microfluidics where laminar flow dominates the system.

\section{Acknowledgments}

We gratefully acknowledge the work of past and present members of our laboratory. This work was partly supported by JSPS Grants-in-Aid for Scientific Research (KAKENHI) (No. 17H02755) and the Japan Keirin Autorace (JKA) Foundation. We thank Ryan Wilkinson, Ph.D., from Edanz Group (https://en-author-services.edanz.com/ac) for editing a draft of this manuscript.

\section{References}

1 G. M. Whitesides: Nature 442 (2006) 368. https://doi.org/10.1038/nature05058

2 R. C. Lo: Chem. Eng. Process Tech. 1 (2013) 1002.

3 R. Daw and J. Finkelstein: Nature 442 (2006) 367. https://doi.org/10.1038/442367a

4 Y. Temiz, R. D. Lovchik, G. V. Kaigala, and E. Delamarche: Microelectron. Eng. 132 (2015) 156. https://doi. org/10.1016/j.mee.2014.10.013

5 D. Beebe, J. S. Moore, J. M. Bauer, Q. Yu, R. H. Liu, C. Devadoss, and B. Jo: Nature 404 (2000) 588. https://doi. org/10.1038/35007047

6 D. E. W. Patabadige, J. Shu, J. Sibbitts, J. Sadeghi, K. A. Sellens, and C. Culbertson: Anal. Chem. 88 (2016) 320. https://doi.org/10.1021/acs.analchem.5b04310

7 D. Stroock, S. K. W. Dertinger, A. Ajdari, I. Mezić, H. A. Stone, and G. M. Whitesides: Science 295 (2002) 647. https://doi.org/10.1126/science.1066238

8 T. Yasui, Y. Omoto, K. Osato, N. Kaji, N. Suzuki, T. Naito, Y. Okamoto, M. Tokeshi, E. Shamoto, and Y. Baba: Anal. Sci. 28 (2012) 57. https://doi.org/10.2116/analsci.28.57

9 D. C. Duffy, H. L. Gillis, J. Lin, N. F. Sheppard, and G. J. Kellogg: Anal. Chem. 71 (1999) 4669. https://doi. org/10.1021/ac990682c

10 J. Park, V. Sunkara, T.-H. Kim, H. Hwang, and Y.-K. Cho: Anal. Chem. 84 (2012) 2133. https://doi.org/10.1021/ ac203163u

11 L. X. Kong, A. Perebikovsky, J. Moebius, L. Kulinsky, and M. Madou: J. Lab. Autom. 21 (2016) 323. https:// doi.org $/ 10.1177 / 2211068215588456$

12 N. R. Glass, R. J. Shilton, P. P. Y. Chan, J. R. Friend, and L. Y. Yeo: Small 8 (2012) 1881. https://doi.org/10.1002/ smll.201102282

13 M. Madou, J. Zoval, G. Jia, H. Kido, J. Kim, and N. Kim: Annu. Rev. Biomed. Eng. 8 (2006) 601. https://doi. org/10.1146/annurev.bioeng.8.061505.095758

14 T. Morijiri, S. Sunahiro, M. Senaha, M. Yamada, and M. Seki: Microfluid Nanofluid 11 (2011) 105. https://doi. org/10.1007/s10404-011-0785-6

15 T. Abe, S. Okamoto, A. Taniguchi, M. Fukui, A. Yamaguchi, Y. Utsumi, and Y. Ukita: Anal. Methods 12 (2020) 4858. 
16 M. Takeuichi, K. Fujitani, A. Ishimoto, A. Yamaguchi, and Y. Utsumi: IEEJ Trans. C 140 (2020) 465. https:// doi.org/10.1541/ieejeiss.140.465

17 D. J. Kinahan, S. M. Kearney, N. A. Kilcawley, P. L. Early, M. T. Glynn, and J. Ducrée: PloS One 11 (2016) e0155545. https://doi.org/10.1371/journal.pone.0155545

18 K. Jahromi, M. Saadatmand, M. Eghbal, and L. P. Yeganeh: Sci. Rep. 10 (2020) 11039. https://doi. org/10.1038/241598-020-67995-3

19 A. Yamaguchi, A. Hirohata, and B. Stadler: Nanomagnetic Materials, Fabrication, Characterization and Application (Elsevier, 2021). ISBN: 978-012-822349-9

20 Roche: https://www.roche.com/ (accessed November 2021).

21 Abaxis: https://www.abaxis.com/ (accessed November 2021).

22 Samsug Healthcare: https://www.Samsunghealthcare.com/en/ (accessed November 2021).

23 Sysmex: https://www.sysmex.co.jp/en/ (accessed November 2021).

24 P. M. C. Lacey: Trans. Inst. Chem. Eng. 21 (1943) 53.

25 Y. Nakata and M. Yamanori: J. Soc. Powder Technol. Japan 54 (2017) 296. https://doi.org/10.4164/sptj.54.296

26 M. Camesasca, M. Kaufman, and I. Manas-Zloczower: Macromol. Theory Simul. 15 (2006) 595. https://doi. org/10.1002/mats.200600037

27 P. S. Fodor and M. Kaufman: Modern Phys. Lett. B 25 (2011) 1111. https://doi.org/10.1142/S0217984911026826

28 https://imagej.net/Fiji/Downloads (accessed November 2021). 